\title{
Proportional and derivative control for steady-state vibration mitigation in a piecewise linear beam system
}

\author{
R.H.B. Fey • R.M.T. Wouters $\cdot$ H. Nijmeijer
}

Received: 18 May 2009 / Accepted: 15 October 2009 / Published online: 5 November 2009

(C) The Author(s) 2009. This article is published with open access at Springerlink.com

\begin{abstract}
A control using Proportional and/or Derivative feedback (PD-control) is applied on a piecewise linear beam system with a flushing one-sided spring element for steady-state vibration amplitude mitigation. Two control objectives are formulated: (1) minimize the transversal vibration amplitude of the midpoint of the beam at the frequency where the first harmonic resonance occurs, (2) achieve this in a larger (low) excitation frequency range, where the lowest nonlinear normal mode dominates the response. Experimentally realizable combinations of PD-control are evaluated for both control objectives. Eventually objective (1) is realized by applying proportional control only, whereas derivative control is selected to realize objective (2). The vibration reduction that is achieved in simulations and validated by experiments is very significant for both objectives. Current results obtained with active PD-control are compared with
\end{abstract}

R.H.B. Fey $(\varangle) \cdot$ H. Nijmeijer

Department of Mechanical Engineering, Eindhoven

University of Technology, P.O. Box 513,

5600 MB Eindhoven, The Netherlands

e-mail: R.H.B.Fey@tue.nl

H. Nijmeijer

e-mail: H.Nijmeijer@tue.nl

R.M.T. Wouters

Department of Technology, YACHT BV, P.O. Box 12610,

1100 AP Amsterdam-Zuidoost, The Netherlands

e-mail: Ron.Wouters@yacht.nl earlier results obtained using a passive dynamic vibration absorber.

Keywords Piecewise linear - Vibration mitigation · Proportional and derivative control

\section{Introduction}

In many situations in structural dynamics, avoidance of resonances is required to prevent malfunctioning, fatigue, or direct breakdown of the structure. First of all, the mechanical design of the structure should be optimized in order to avoid resonances. However, despite high efforts in the design stage, the occurrence of resonances cannot always be avoided, or the level of the occurring vibration amplitudes still may be too high. In these cases, depending on the specific requirements for the problem under consideration, passive, semiactive, or active control may be applied to mitigate the vibration levels. Passive control in general is robust and is achieved by adding mechanical elements to the structure. Examples are dynamic vibration absorbers and constrained layer dampers [1-4]. In semiactive control, the parameter values of certain structural elements are controlled as a function of time [5]. In active control [6-9], actuators are used to apply time dependent loads on the structure to attenuate the vibration levels. The references mentioned above focus on linear structures with linear control. 
This paper focuses on active control for steadystate vibration mitigation of a periodically excited, archetype piecewise linear system, consisting of a linear beam, which is supported by a one-sided linear elastic spring. The uncontrolled steady-state behavior of this type of system was studied numerically as well as experimentally in [10]. The steady-state behavior observed in this system is representative for the behavior observed in many piecewise linear systems met in engineering practice, which shows the relevance of this work. Five practical examples of these systems are: (1) a mooring buoy near an oil platform, to which a ship is attached via a cable, which periodically slackens due to sea wave excitation [11], (2) folded solar array panels, which are attached to a satellite during launch, and which are separated from each other by one-sided spring elements [12], (3) bridge hangers in a suspension bridge [13], (4) a piecewise linear pantograph current collector suspension system [14, 15], and (5) a bump stop in a wheel suspension system [16].

Depending on the amount of nonlinearity and damping, the steady-state behavior of controlled and uncontrolled piecewise linear systems may be highly varying $[10,11,17-22]$. In general, next to harmonic resonances, also super and subharmonic resonances may occur. Moreover, in usually small excitation frequency intervals, also quasi-periodic or chaotic responses/resonances may exist. Resonance peaks are straight up for the case, that the one-sided spring flushes to the remainder of the system in the static equilibrium position $[10,11,17,18]$. However, in piecewise linear systems, resonance peaks may also show hardening behavior [12, 23, 24], due to bridging of the backlash between system and the one-sided spring, or softening behavior [12, 23], due to loss of initial pretension between the system and the onesided spring.

The objective of this paper is to investigate, to what extent relatively simple Proportional and/or Derivative feedback control (PD-control) can be applied, in order to reduce steady-state vibrations/resonances in a piecewise linear beam system, where a one-sided spring flushes to a linear beam in the static equilibrium position. In this paper, the steady-state vibrations in the uncontrolled system will be caused by a disturbing harmonic excitation force. Two different control objectives will be considered. The first objective will be to suppress the 1st harmonic resonance peak at the corresponding resonance frequency. The second objective will be to reduce the vibration levels of the system in a wider frequency range. In this frequency range, the first nonlinear normal mode should still be dominant, however. This requirement in fact justifies attempting the PD-control approach. The two control objectives will be formulated in more detail later. The same two control objectives were also posed in [25] with respect to the same piecewise linear beam system. In [25], the objectives were successfully realized by means of a passive linear Dynamic Vibration Absorber (DVA).

Obviously, application of more advanced (nonlinear) controllers and observers may result in increased vibration reduction, but may also result in increased costs, increased complexity, and lower reliability. In $[26,27]$, for a similar piecewise linear system, resonance/vibration attenuation is realized in a wider frequency band, using an observer-based state feedback controller. In [28, 29], again for a similar, periodically excited, piecewise linear beam system, partial feedback linearization was used. However, in [28, 29], the control objective was different compared to the control objectives of the current paper. For a specific excitation frequency, the uncontrolled piecewise linear system shows a stable $1 / 2$ subharmonic resonance coexisting with an unstable harmonic solution with low amplitude. In $[28,29]$, the control objective was to control the $1 / 2$ subharmonic solution to the harmonic solution and to stabilize this solution.

This paper is organized as follows. First, in the next section, the experimental setup of the piecewise linear beam system will be introduced. The steady-state behavior of the uncontrolled system will be discussed in Sect. 3. In Sect. 4, the motivation to use PD-control will be discussed further, two control objectives will be formulated, PD-controller constraints will be discussed, and the PD controller design approach will be presented. Section 5 will discuss the simulation model of the system. In Sect. 6, first the separate effect of proportional feedback, and secondly the separate effect of derivative feedback on the steady-state behavior of the closed loop system will be investigated. Experimental and numerical results will be compared. Based on the insights obtained, in Sect. 7, two PD-control settings will be determined in order to realize the two control objectives as good as possible. In Sect. 8, results obtained in this paper and results obtained in [25] using a DVA will be compared. Finally, in Sect. 9, conclusions will be drawn and recommendations for future research will be given. 


\section{Experimental setup}

Figure 1 shows the schematic representation of the experimental setup of the system in the horizontal plane. The influence of gravity is negligible. The setup exists of a steel main beam (a), which is supported at each end by a leaf spring (b). In the middle of the beam, a one-sided leaf spring (c) is placed flushing to the main beam (a) making the system piecewise linear. This means that the one-sided spring neither introduces backlash nor pretension in the static equilibrium situation. The backlash, which is seen in Fig. 1, is shown for sketching purposes only, to make clear that the leaf spring is indeed a one-sided spring. The contact force between spring (c) and the main beam will become nonzero for a displacement of the midpoint of beam (a) in the negative $y$-direction; see Fig. 1. The amount of nonlinearity in the piecewise linear system may be indicated by the ratio of the stiffness of the one-sided spring and the transversal linear stiffness of the main beam (a), halfway its length. Here, this ratio equals $\alpha=2.7[-]$.

The system is transversally excited by a disturbing harmonic force generated by an eccentrically rotating mass mechanism, which is attached to the middle of beam (a) and driven by an electric synchronous motor. The excitation frequency $f=\omega /(2 \pi)$ will be varied between 10 and $60[\mathrm{~Hz}]$.

The actuator, which exerts the PD-control force to the system, is placed as near as possible $(0.2 \mathrm{~m})$ to the midpoint of the beam. The operation of this actuator is based on the principle that a force is generated, when a current flows through a coil, which is placed in a permanent magnetic field. The midpoint transversal displacement and acceleration are measured using an LVDT and an accelerometer, respectively. The measured signals are processed by a data acquisition system, which determines an appropriate current amplifier input. Subsequently, the amplified input is offered to the digital PD-controller. In order to determine the PD control force of the actuator, next to the displacement signal, actually the velocity signal is needed. The velocity signal is obtained by filtering the measured acceleration signal by a first order, high pass, analog filter (filter frequency: $7.2 \mathrm{~Hz}$ ), and subsequent ana$\log$ integration of the filtered acceleration signal over time. This high pass filter is used to avoid drift. Then the analog velocity signal is digitized and a second order, low pass, Butterworth filter (filter frequency: $170 \mathrm{~Hz}$ ) is applied to suppress high-frequency noise. As a side-effect, this will also delete deterministic high-frequency components in the velocity signal.

\section{Steady-state behavior of the uncontrolled system}

In Fig. 2, the steady-state response of the uncontrolled system is shown. In this figure, the quantity "max disp" of a steady-state solution of the transversal displacement of the beam midpoint $y_{\text {mid }}(t)$, defined by

$\max \operatorname{disp}=\max y_{\text {mid }}(t)-\min y_{\text {mid }}(t)$,

is determined for excitation frequencies $f$ ranging from 10 to $60[\mathrm{~Hz}]$. Note that a value of max disp close
Fig. 1 Schematic representation of the experimental setup

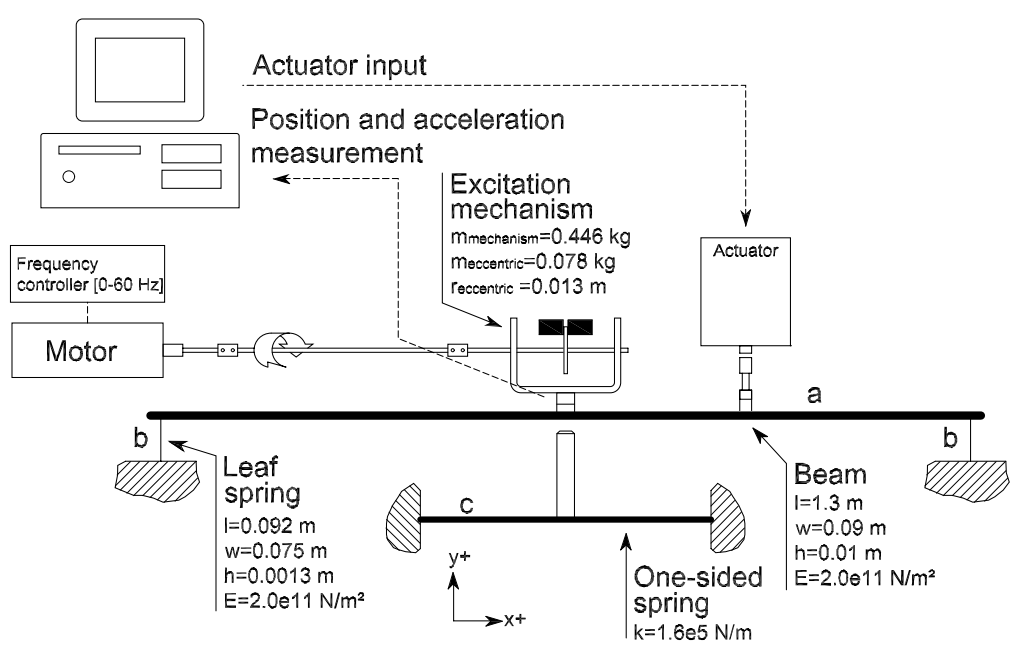




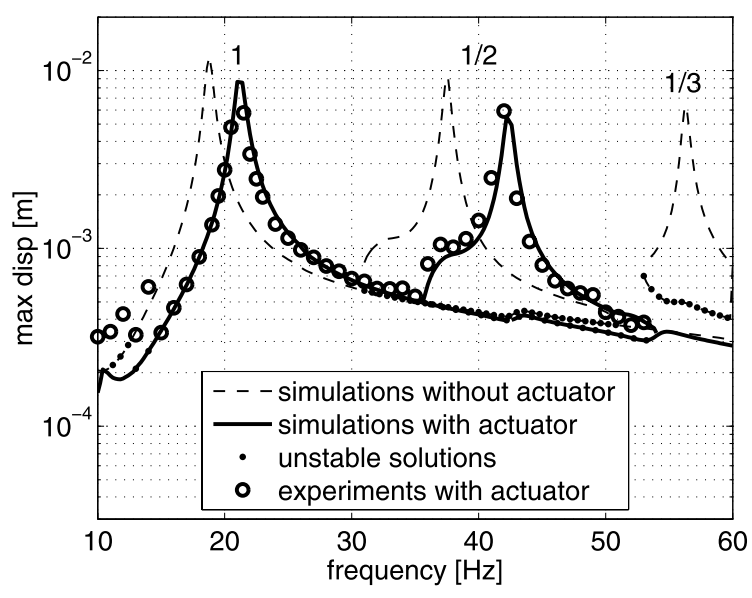

Fig. 2 Response of the uncontrolled system

to zero does not necessarily mean that the overall vibration level of the beam is close to zero, because the beam may be vibrating in a shape with a node near or in the midpoint of the beam.

Figure 2 shows both simulation results and experimental results. Simulation results are based on a numerical model, which will be introduced in Sect. 5, and are given for the uncontrolled system without actuator as well as the uncontrolled system with passive actuator dynamics. In the former case, stable periodic solutions are indicated with dashed lines. In the latter case, the stable periodic solutions are indicated with solid lines. In both cases, unstable periodic solutions are indicated with black dots. For clarity, the uncontrolled system with passive actuator dynamics is the system including the inertia, damping, and elastic properties of the actuator. The actuator control force, however, is still equal to zero.

Calculation of branches of periodic solutions and their stability and detection of bifurcation points on these branches is based on theory and numerical methods described in, for example [17, 30, 31]. Although the forces occurring in the system are continuous, the current system has a discontinuity in the stiffness. In numerical simulations, this should be accounted for, for example, by means of event detection, to guarantee accurate dynamic responses [32, 33].

Experimental results, indicated by circles, are only included for the uncontrolled system with passive actuator dynamics. A good correspondence can be observed between experimental and simulation results.

Figure 2 shows that for the case with as well as for the case without passive actuator dynamics, a harmonic resonance peak occurs near $20[\mathrm{~Hz}]$, and a related $1 / 2$ subharmonic resonance near the double of this frequency. For the case without actuator also a related $1 / 3$ subharmonic resonance is visible near $56[\mathrm{~Hz}]$. This $1 / 3$ subharmonic resonance occurs above $60[\mathrm{~Hz}]$ for the case with passive actuator dynamics. Thus, by adding the passive actuator dynamics, the global dynamic behavior of the uncontrolled system does not change. Resonance peaks shift to somewhat higher excitation frequencies.

\section{PD-control: motivation, control objectives, constraints, and design}

Apart from the fact that it is easy to implement, the motivation to attempt a PD-control approach to mitigate the vibration level in this piecewise linear system originates from the observation that, in the frequency range of interest (10-60 [Hz]), actually only the lowest nonlinear normal mode $[19,34,35]$ is dominant. It is important to realize this. Namely, for linear multidegree of freedom systems, it is well known that a single PD-controller in principle is capable of controlling only one single normal mode. Therefore, in the case of the current piecewise linear system, the best one may hope for is that the PD-controller can control, more or less, one single nonlinear normal mode. In [25], a passive linear dynamic vibration absorber was capable to mitigate the vibration level in this piecewise linear system in the frequency range $10-60[\mathrm{~Hz}]$, being the dominant frequency range of the first nonlinear normal mode. This may be seen as an indication that the PD-controller can be successfully applied here too. In Sect. 5, the eigenmode of the system without the one-sided spring, which is closely related to the first nonlinear normal mode will be shown.

As announced in the Introduction already, now two separate control objectives are formulated:

Control objective 1 Minimize "max disp" defined by (1) at the first harmonic resonance frequency of $21.2[\mathrm{~Hz}]$ of the uncontrolled piecewise linear beam system with passive actuator dynamics; see Fig. 2.

Control objective 2 Reduce "max disp" defined by (1) in the frequency range $10-60[\mathrm{~Hz}]$. No mathematical optimization criterion will be used to realize this objective. Here, the performance of the PD-control ac- 
tion is evaluated by visual inspection (tuning) of multiple amplitude-frequency plots such as Fig. 2. In this visual inspection, the focus will mainly be on the success of the suppression of the harmonic and subharmonic resonance peaks, which are present in the uncontrolled situation. Obviously, simultaneously the appearance of new resonance peaks in the frequency range 10 $60[\mathrm{~Hz}]$ is prohibited. This visual inspection will be carried out for all experimentally realizable settings of the two PD control parameters $k_{\mathrm{p}}$ and $k_{\mathrm{d}}$, which will be introduced later.

It is emphasized again that vibration reduction of the midpoint of the beam does not guarantee overall vibration reduction of the beam because for the controlled situation this midpoint may behave as a node, while the rest of the beam is still vibrating at high vibration levels. For every combination of the control parameters, $k_{\mathrm{p}}$ and $k_{\mathrm{d}}$, it will be checked afterwards, if the latter situation, which obviously is undesirable with respect to overall vibration reduction, does not occur.

Obviously, the beam midpoint would have been the ideal controller position, because this is the position:

(1) where the disturbing harmonic excitation of the system takes place,

(2) for which the control objectives are formulated,

(3) where the one-sided spring exerts its force to the beam, and

(4) where the dominant nonlinear normal mode shows a maximal transversal displacement.

Actually, a controller located at the beam midpoint position, which could counterbalance the harmonic excitation force, would cancel all beam vibrations. However, in many practical situations, it is not possible to apply the control force on the ideal location. This is also the case in our experimental set-up, where the control force is applied $0.2[\mathrm{~m}]$ from the midpoint of the beam, which is the position as near as possible to the beam midpoint from a practical point of view.

The digital PD-controller, which makes use of the measured transversal displacement and the measured transversal acceleration of the midpoint of the beam (recall that the acceleration is filtered and integrated over time), determines the magnitude of the PD-control force $F_{\mathrm{c}}$, which should be equal to:

$F_{\mathrm{c}}=-k_{\mathrm{p}} y_{\mathrm{mid}}-k_{\mathrm{d}} \dot{y}_{\mathrm{mid}}$ where $y_{\text {mid }}$ and $\dot{y}_{\text {mid }}$ are respectively the beam's midpoint transversal displacement and velocity. The PDcontroller set-point consists of the corresponding two gains, $k_{\mathrm{p}}$ and $k_{\mathrm{d}}$, respectively. In the experimental setup, the following constraints apply to $k_{\mathrm{p}}$ and $k_{\mathrm{d}}$ :

$-6 \times 10^{4} \leq k_{\mathrm{p}} \leq 2.5 \times 10^{4}[\mathrm{~N} / \mathrm{m}]$,

$0 \leq k_{\mathrm{d}} \leq 600[\mathrm{Ns} / \mathrm{m}]$

A negative value of $k_{\mathrm{p}}$ will decrease the resonance frequencies, because the effective stiffness of the controlled system will decrease. The lower bound on the proportional gain $k_{\mathrm{p}}$ is set to $-6 \times 10^{4} \mathrm{~N} / \mathrm{m}$, because lower values will result in negative effective stiffness, and consequently unstable behavior. The upper bound on the proportional gain $k_{\mathrm{p}}$ is $+2.5 \times 10^{4} \mathrm{~N} / \mathrm{m}$. A higher value of $k_{\mathrm{p}}$ will make the system unstable due to unacceptable measurement noise amplification in the feedback loop. The derivative gain $k_{\mathrm{d}}$ influences the effective damping in the system. The structural damping in the uncontrolled system is very low. Therefore, the lower bound on the derivative gain $k_{\mathrm{d}}$ is set to $0 \mathrm{Ns} / \mathrm{m}$, because negative values of $k_{\mathrm{d}}$ will quickly make the system unstable when the effective damping becomes negative. The upper bound on the derivative gain is $600 \mathrm{Ns} / \mathrm{m}$. A higher value again will cause unacceptable measurement noise amplification. Larger values of $k_{\mathrm{d}}$ experimentally do not result in vibration reduction and lead to increased deviations between experimental and theoretical response amplitudes.

To realize each separate control objective, in Sect. 7, two different set-points of the PD-controller will be determined by evaluating the steady-state response for a large number of PD-controller set-points satisfying the constraints given by (3). Prior to this, first the simulation model of the system with and without control will be discussed in the next section and the effects of separate P-action and separate D-action on the system's behavior will be determined in Sect. 6 .

\section{Simulation model}

In order to avoid unacceptably high CPU-times, a reduced 4 degrees of freedom model has been derived, which accurately approximates the dynamic behavior of the piecewise linear system with PD-control in the frequency interval of interest $(10-60[\mathrm{~Hz}])$ :

$M \ddot{x}+D \dot{x}+K\left(y_{\text {mid }}\right) x=F$, 
where:

$$
\begin{aligned}
& x=\left[\begin{array}{llll}
y_{\text {mid }} & y_{\text {act }} & p_{2} & p_{3}
\end{array}\right]^{T}, \\
& M=T^{T} M_{\mathrm{o}} T, \quad D=T^{T} D_{\mathrm{o}} T, \\
& K_{1}=T^{T} K_{\mathrm{o}} T, \quad F=T^{T} F_{\mathrm{o}}, \\
& K\left(y_{\text {mid }}\right)=\left\{\begin{array}{ll}
K_{1} & \text { if } y_{\text {mid }}>0 \\
K_{1}+K_{\mathrm{os}} & \text { if } y_{\text {mid }} \leq 0
\end{array}, \quad\right. \text { and }
\end{aligned}
$$

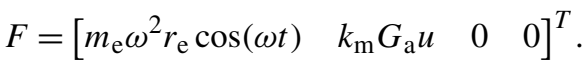

Matrices $M, D$, and $K_{1}$ represent respectively the reduced mass, reduced damping, and reduced stiffness matrix, derived by dynamic reduction of the original linear finite element model. Matrices $M_{\mathrm{o}}, D_{\mathrm{o}}$, and $K_{\mathrm{o}}$ are respectively the mass, damping, and stiffness matrix of the original finite element model, containing the inertial, damping, and elastic properties of the main beam plus end supports, the passive actuator dynamics, and the periodic excitation mechanism. Columns $F$ and $F_{\mathrm{O}}$ are respectively the reduced column and the original column with external forces. Both columns contain two nonzero elements representing the harmonic excitation force and the control force.

The original displacement field $q$ of the unreduced finite element model is related to the column with reduced degrees of freedom $x$ by means of the following approximation based on the Ritz reduction matrix $T$ :

$q=T x$.
The Ritz reduction matrix $T$ is based on 4 modes, which are derived from the original undamped finite element model without the one-sided spring, namely: the 2nd eigenmode $\varphi_{2}$ and the 3 rd eigenmode $\varphi_{3}$, and two residual flexibility modes $[17,36]$, which are defined for $y_{\text {mid }}$ and $y_{\text {act }}$, the transversal displacements of respectively the beam midpoint and the actuator position. The 2nd eigenmode $\varphi_{2}$, with corresponding eigenfrequency $f_{2}=16.2[\mathrm{~Hz}]$ and generalized degree of freedom $p_{2}$, and the 3 rd eigenmode, with corresponding eigenfrequency $f_{3}=54[\mathrm{~Hz}]$ and generalized degree of freedom $p_{3}$, are solutions of the undamped eigenvalue problem:

$\left(-\left(2 \pi f_{\mathrm{k}}\right)^{2} M_{\mathrm{o}}+K_{\mathrm{o}}\right) \varphi_{\mathrm{k}}=0$.

The two residual flexibility modes are static correction modes, which guarantee unaffected static load behavior for the reduced model.

Figure 3 shows the lowest four eigenmodes $\varphi_{1}, \varphi_{2}$, $\varphi_{3}, \varphi_{4}$ of the undamped eigenvalue problem, given by (10), with the corresponding eigenfrequencies. In the experimental set-up, the 1st eigenmode is suppressed by the drive shaft of the excitation mechanism, so it does not need to be included in the reduced model. The 4th eigenfrequency exceeds the frequency range of interest to a large extent. Note that the passive actuator dynamics slightly disturb the (anti)symmetry of eigenmodes with respect to the beam midpoint.

The lowest eigenfrequency of the one-sided spring (see Fig. 1) is much higher than the frequency range
Fig. 3 First 4 eigenmodes (black solid lines) following from (10). Dashed lines indicate the undeformed structure. The midpoint position is indicated by $\square$; the actuator position is indicated by $\triangle$. For presentational reasons, horizontal and vertical directions are sometimes scaled differently
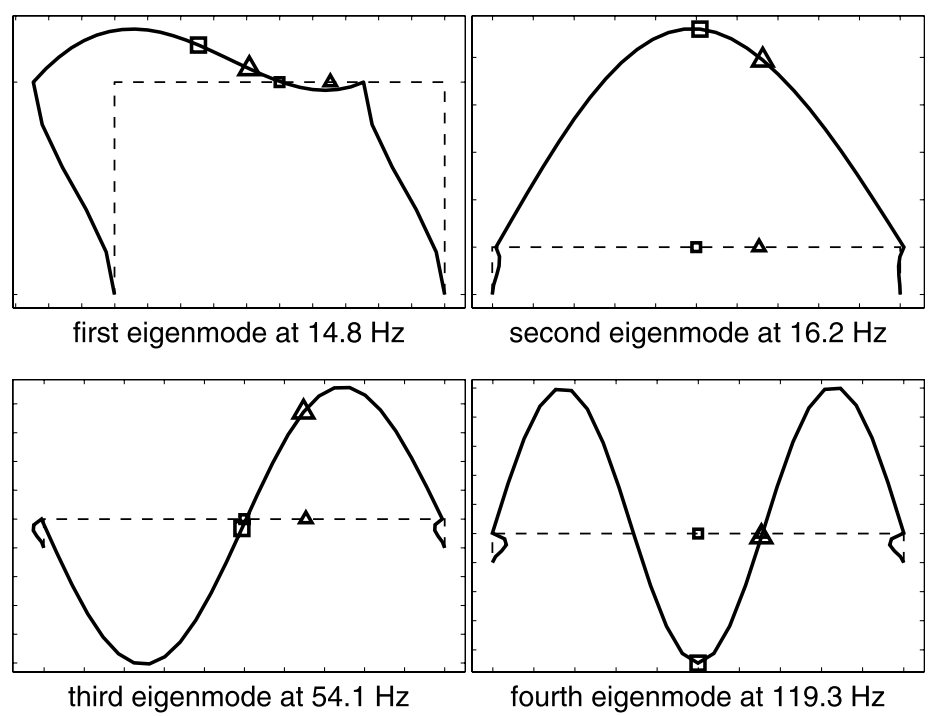
of interest (10-60 [Hz]). Therefore, the inertia of the one-sided spring may be neglected. In (7), the stiffness of the one-sided spring is represented by matrix $K_{\mathrm{OS}}$ containing one nonzero entry $(1,1)$ equal to $k_{\mathrm{os}}$.

Damping matrix $D_{\mathrm{o}}$ is based on approximately $2 \%$ modal damping for each mode and additionally contains the discrete viscous damping constant of the actuator. The $2 \%$ modal damping among others accounts for energy losses due to impacts and results in a good match between measured and calculated resonance amplitudes.

In (8), entries 1 and 2 of column $F$ contain respectively the harmonic excitation force and the control force $F_{\mathrm{c}}$. In the harmonic excitation force, $m_{\mathrm{e}}$ is the rotating eccentric mass, $r_{\mathrm{e}}$ its eccentricity, and $\omega=2 \pi f$ its angular frequency, where $f$ is the excitation frequency in $[\mathrm{Hz}]$. In the actuation force, $k_{\mathrm{m}}$ is the motor constant, $G_{\mathrm{a}}$ the gain of the current amplifier, and $u$ the applied voltage. This voltage is chosen in such a manner that control force $F_{\mathrm{c}}$ according to (2) is realized. The uncontrolled situation is represented by $u=0[\mathrm{~V}]$.

In the numerical analysis, the transversal velocity signal of the midpoint of the beam is directly available in contrast to the experimental situation. The low-pass Butterworth filter, which is used in the experiments in order to reduce high frequency noise (see the end of Sect. 2) is also applied to this velocity signal in the simulations. As a result, also in the numerical analysis the deterministic high-frequency components in the velocity signal are removed to mimic the experimental situation.

Note that the nonlinear normal mode, closely related to the second eigenmode of the linear system with eigenfrequency $f_{2}=16.2[\mathrm{~Hz}]$, dominates the response near the first harmonic resonance peak at $21.2[\mathrm{~Hz}]$ in Fig. 2. In fact, it dominates almost the whole frequency range of $10-60 \mathrm{~Hz}$. This peak occurs at $21.2[\mathrm{~Hz}]$ instead of $16.2[\mathrm{~Hz}]$ due to the presence of the one-sided spring. The value of $21.2[\mathrm{~Hz}]$ can be checked in good approximation by using the following expression for the resonance frequency of an undamped, piecewise linear, single degree of freedom system, which is derived in [18] for the case of flush:

$$
f_{\mathrm{pwl}}=\frac{2 \sqrt{1+\alpha}}{1+\sqrt{1+\alpha}} f_{\text {lin }} .
$$

ln (11), $\alpha$ is the ratio between the stiffness of the one-sided spring and the stiffness of the linear spring and $f_{\text {lin }}$ is the eigenfrequency of the linear, single degree of freedom, mass-spring system. As stated before, in our case, the ratio between the stiffness of the one-sided spring and the transversal stiffness of the beam, halfway the length of the beam, is $\alpha=$ 2.7 [-]. Substituting this value and the value of $f_{\text {lin }}=$ $f_{2}=16.2[\mathrm{~Hz}]$ in (11) results in $f_{\mathrm{pwl}}=21.3[\mathrm{~Hz}]$, which is, as expected, slightly above the experimentally observed resonance frequency of $21.2[\mathrm{~Hz}]$ for our weakly damped beam system.

\section{Effects of separate P-action and separate D-action}

In this section, the effects of separate P-control and separate D-control on the steady-state behavior of the piecewise linear beam system will be investigated. With the insights obtained in this way, combined PDcontrol in Sect. 7 can be understood better.

First, the effect of P-action on the response of the piecewise linear system is investigated $\left(k_{\mathrm{d}}=\right.$ $0[\mathrm{Ns} / \mathrm{m}]$ ). Figure 4 shows max disp (see (1)) in the excitation frequency range 10-60 [Hz] for several values of $k_{\mathrm{p}}$. Stable simulation results are validated by experimental results (circles). For larger gains, discrepancies between numerical and experimental results increase somewhat because the influence of the measurement noise in the feed-back loop increases. Increasing $k_{\mathrm{p}}$ shifts the resonances to higher frequencies because "stiffness" is added to the system.

Obviously, the $1 / 2$ subharmonic resonance near $42[\mathrm{~Hz}]$ shifts approximately twice as much as the related harmonic resonance near 21 [Hz]. In Fig. 4, only positive values of $k_{\mathrm{p}}$ are considered. However, negative values may also be applied and in fact will be used in Sect. 7.

Figure 5 shows the effect of only D-action $\left(k_{\mathrm{p}}=\right.$ $0[\mathrm{~N} / \mathrm{m}])$. Again the experimental and simulation results match well. Derivative feedback significantly suppresses both the harmonic and 1/2 subharmonic resonances near respectively $21[\mathrm{~Hz}]$ and $42[\mathrm{~Hz}]$.

In the experiments, which were all started from rest, a slow frequency sweep up did not reveal other stable steady-state solutions than a slow frequency sweep down, for the considered frequency range. Obviously, 
Fig. 4 Max disp in the range $10-60[\mathrm{~Hz}]$ for varying $k_{\mathrm{p}}[\mathrm{N} / \mathrm{m}]$. Surface: simulation results, unstable numerical solutions are indicated by black dots. Circles: experimental results
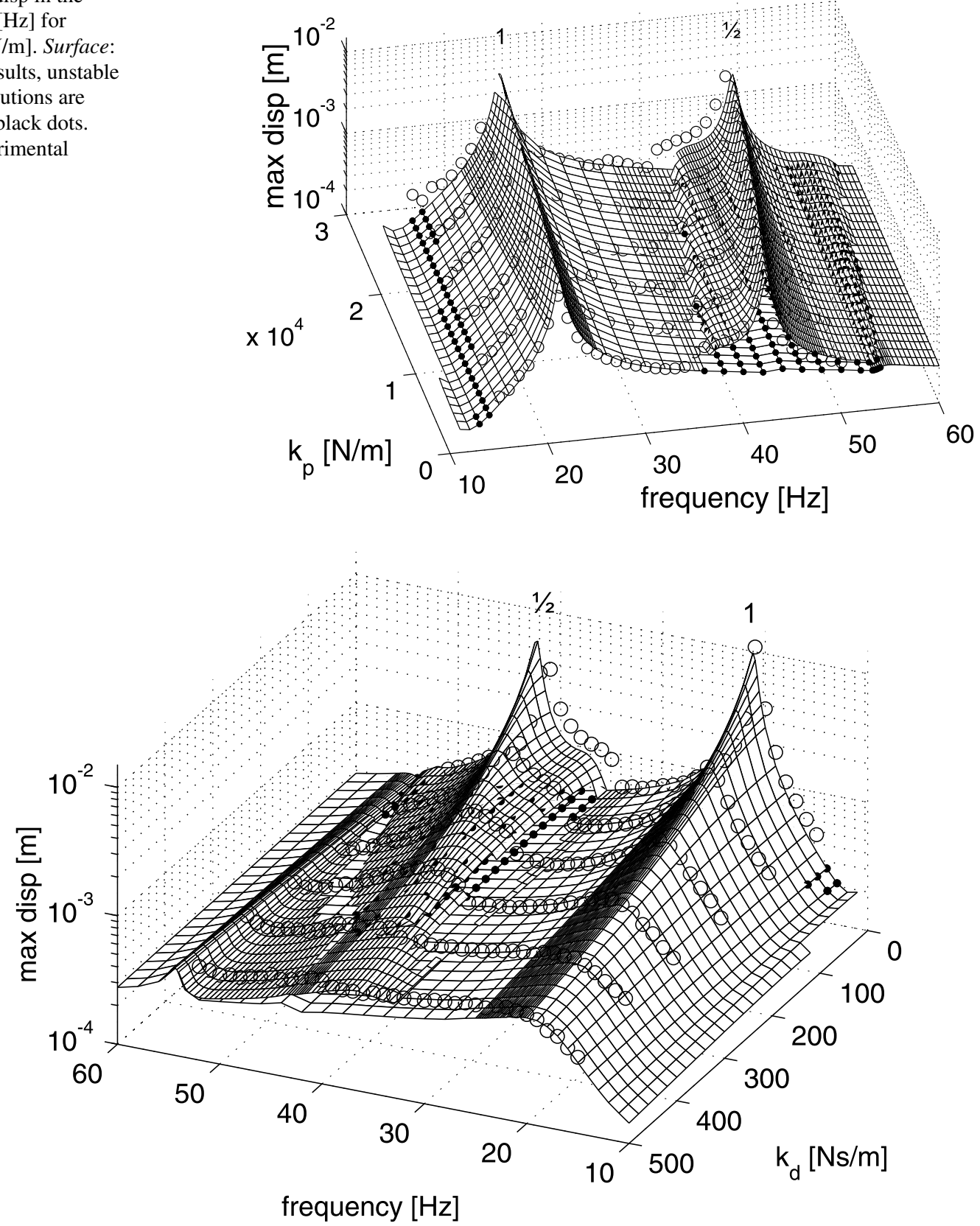

Fig. 5 Max disp in the range 10-60 Hz for varying $k_{\mathrm{d}}[\mathrm{Ns} / \mathrm{m}]$. Surface: simulation results, unstable numerical solutions are indicated by black dots. Circles: experimental results

this does not mean that other stable steady-state solutions, which may have a periodic, quasi-periodic, or chaotic nature, may not (co)exist. For example, by using other initial conditions, or by applying a sudden disturbance to the system, other coexisting stable steady-state solutions may be found.

\section{PD-control: results}

In this section, keeping in mind the effects of using separate P-action and separate D-action, as discussed in Sect. 6, the effect of combined P- and D-action on the system's steady-state behavior will be studied. Ob- 
Fig. 6 max disp in [mm]

for $k_{\mathrm{p}}, k_{\mathrm{d}}$ combinations

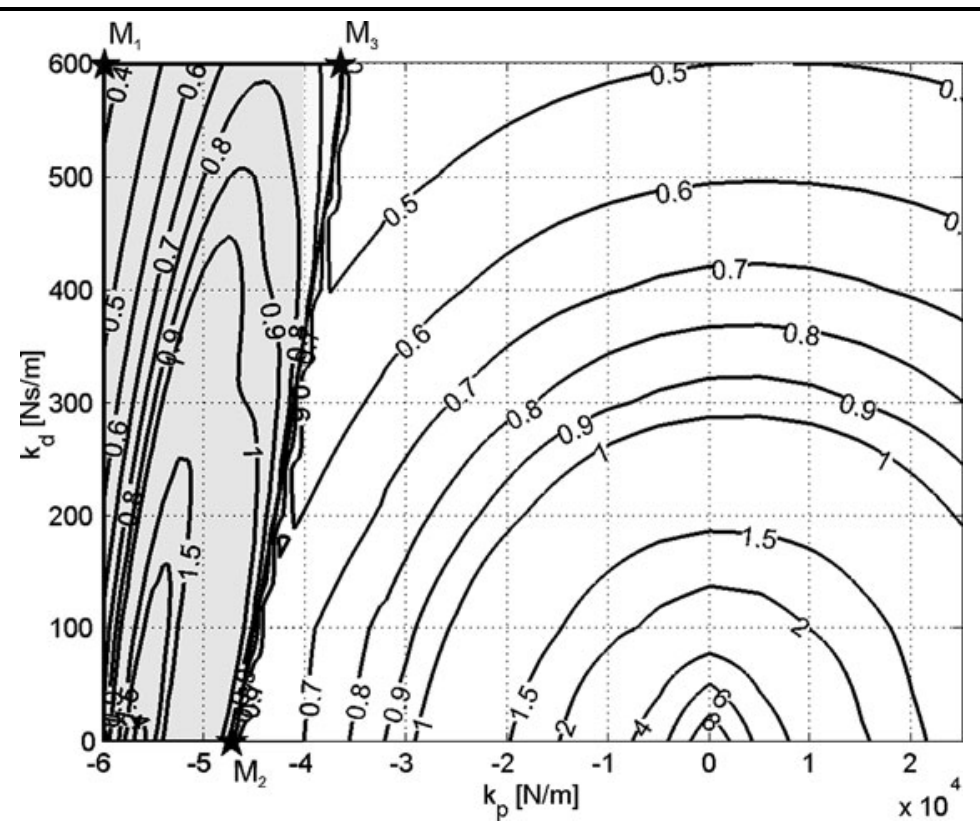

viously, the control objectives will be subjected to the constraints given by (3). The results for control objective 1 and control objective 2 will be discussed in respectively Sects. 7.1 and 7.2. In addition, Sect. 7.3 will discuss some numerical results for the case of collocated control: the control force will be exerted in the middle of the beam. This is done in order to investigate, for both control objectives, how the results will change, if the location of the controller force is changed somewhat.

\subsection{Control objective 1}

Figure 6 shows a contour plot of max disp in [mm] (see (1)) of simulated stable periodic solutions, for all possible $k_{\mathrm{p}}, k_{\mathrm{d}}$ combinations, at an excitation frequency of $21.2[\mathrm{~Hz}]$. The contours in the white area refer to stable harmonic solutions. The contours in the grey area refer to stable $1 / 2$ subharmonic solutions. The boundary between the white area and the grey area consists of period doubling bifurcation points. Set-point $\mathrm{M}_{2}$ $\left(k_{\mathrm{p}}=-4.6 \times 10^{4}[\mathrm{~N} / \mathrm{m}], k_{\mathrm{d}}=0[\mathrm{Ns} / \mathrm{m}]\right)$ is chosen to fulfill control objective 1 because then neither $k_{\mathrm{p}}$ nor $k_{\mathrm{d}}$ needs to be to set to its limit value. Compared to set-points $\mathrm{M}_{1}$ and $\mathrm{M}_{3}$, only a slightly higher max disp value $(0.57 \mathrm{~mm})$ is found in set-point $\mathrm{M}_{2}$. This value is still much lower compared to the value in the uncontrolled situation (about $10[\mathrm{~mm}]$ ).
Figure 7 shows the results for set-point $\mathrm{M}_{2}$ in a wider frequency range. The controlled response shows that, due to the negative proportional feedback, the first harmonic and 1/2 subharmonic resonances are shifted to lower frequencies in such a way, that a local minimum of the maximum displacement of the stable solutions occurs in between at $21.2[\mathrm{~Hz}]$, at the period doubling bifurcation point.

For set-point $\mathrm{M}_{2}$ at $21.2[\mathrm{~Hz}]$, the transversal vibration level at other locations on the beam appears to be of the same order as for the transversal vibrations, halfway the length of the beam, as shown in Fig. 7.

\subsection{Control objective 2}

When the results of all considered combinations of Pand D-action are visually compared (these results are not presented here), it appears that actually only Daction is needed to maximize overall displacement reduction in the range 10-60 [Hz]. Very large P-action could shift all resonances to frequencies above 60 [Hz] but the required $k_{\mathrm{p}}$ is outside the constraint interval given by (3). For the required $k_{\mathrm{p}}$ the amplified measurement noise would result in an unstable system.

The circles in Fig. 8 show the experimental results for the experimentally maximum applicable D-action $\left(k_{\mathrm{p}}=0[\mathrm{~N} / \mathrm{m}], k_{\mathrm{d}}=600[\mathrm{Ns} / \mathrm{m}]\right)$. Both the harmonic and the $1 / 2$ subharmonic resonance peaks are reduced. 
Fig. 7 Vibration reduction of the harmonic resonance of the uncontrolled system at 21.2 [Hz] for set-point $\mathrm{M}_{2}$. Solid line: stable simulation results, black dots: unstable simulation results. Circles: experimental results. Dashed line: response of uncontrolled system including the passive actuator

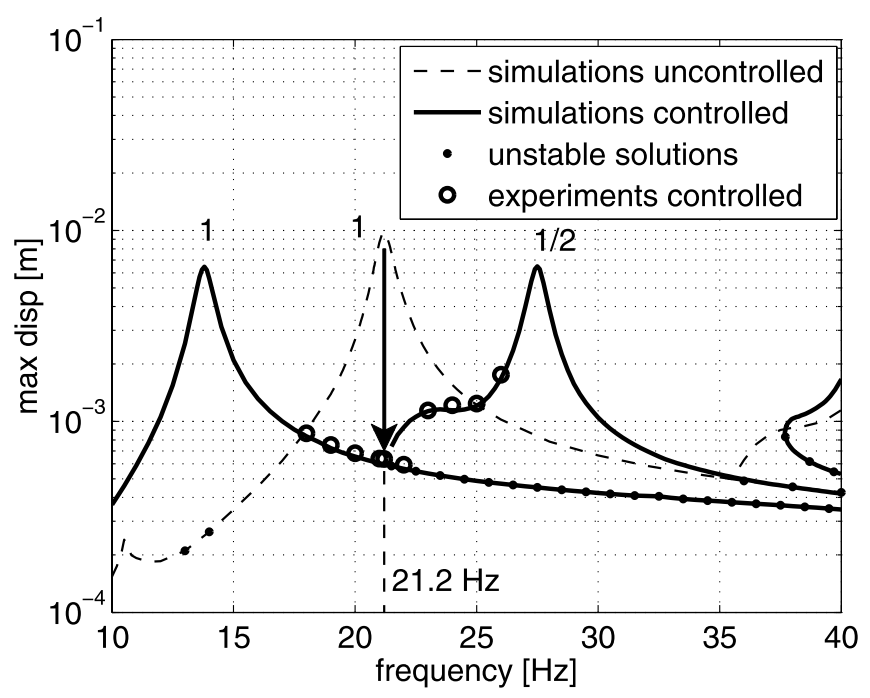

Fig. 8 Vibration reduction in the range $10-60$ [Hz]. Solid line: simulation results. Circles: experimental results (set-point: $k_{\mathrm{p}}=0[\mathrm{~N} / \mathrm{m}]$, $\left.k_{\mathrm{d}}=600[\mathrm{Ns} / \mathrm{m}]\right)$. Dashed line: uncontrolled case. Black dots: unstable numerical solutions of the uncontrolled case

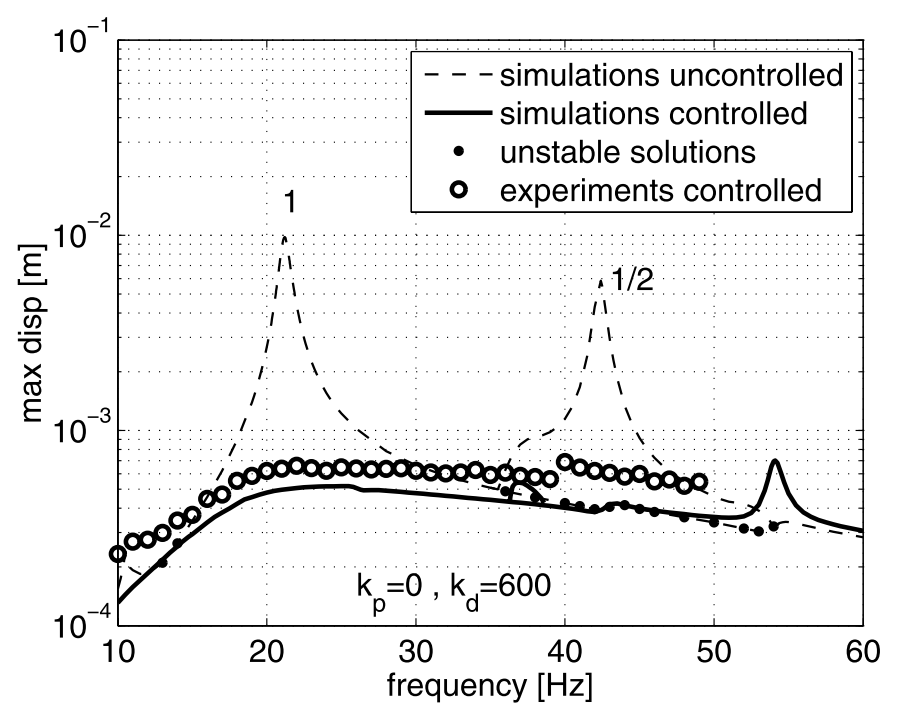

The largest vibration reduction of a factor 20 is observed at the harmonic resonance peak.

The difference between the simulation results and the experimental results in Fig. 8 is due to the noise in the "measured" velocity signal (actually the integrated measured transversal acceleration of the beam midpoint). This results in a limited accuracy of the applied control force. Better correspondence between experimental and simulation results is obtained for lower D-action at the cost of somewhat larger max disp values.

In a very small frequency range near $54[\mathrm{~Hz}]$, the vibration level actually increases somewhat for the controlled situation compared to the uncontrolled sit- uation as presented in Fig. 2. This can be understood when realizing that the control force increases the excitation of the second nonlinear normal mode, which is very closely related to the 3rd linear eigenmode, which is shown in Fig. 3 and has an eigenfrequency of $f_{3}=54[\mathrm{~Hz}]$. It is only due to the passive dynamics of the actuator in the uncontrolled situation that this eigenmode has a node, which not exactly coincides with the beam midpoint. Without the passive dynamics of the actuator, the piecewise linear spring would not have any influence on the eigenmode at $54[\mathrm{~Hz}]$ : the second nonlinear normal mode would convert to a linear eigenmode. 
Fig. 9 Collocated control: max disp in [mm] for $k_{\mathrm{p}}, k_{\mathrm{d}}$ combinations

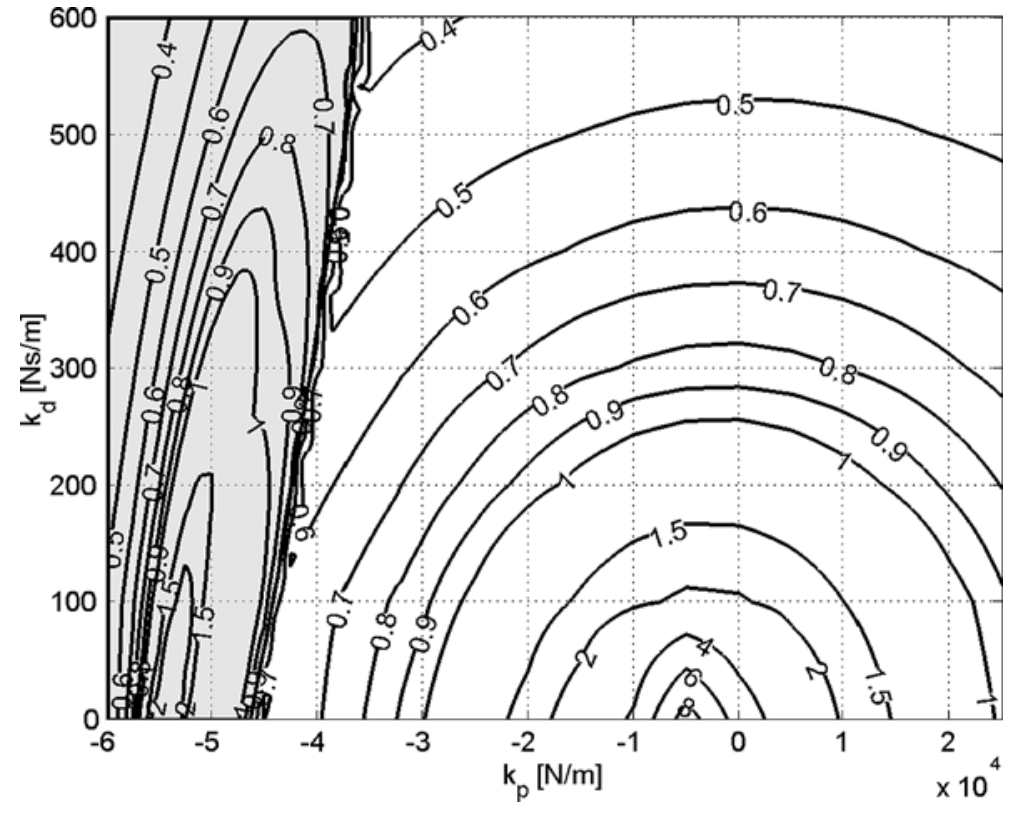

As stated before, the vibration reduction, which is realized for the transversal displacement of the midpoint of the beam, does not guarantee vibration reduction for transversal displacements of other positions on the beam. However, by visual inspection it was verified that also on other beam positions reduction of transversal vibrations is achieved, except for excitation frequencies near the second harmonic resonance peak, i.e., near $54[\mathrm{~Hz}]$.

\subsection{Collocated control}

In the case of collocated control, the transversal displacement and acceleration are again measured in the middle of the beam as before. The control force, however, is now also exerted in the middle of the beam, instead of $0.2 \mathrm{~m}$ outside the middle of the beam as before. For collocated control, Figs. 9 and 10 show the results for respectively control objective 1 and control objective 2 . These figures are to be compared with the results shown earlier in respectively Figs. 6 and 8 .

Repositioning of the PD controller to the middle of the beam obviously means that the inertia, damping, and stiffness of the inactive actuator, i.e., its passive dynamic properties, are moved to the middle position of the beam. This implies a change in the dynamic properties of the uncontrolled system. This can be seen, for example, when comparing Fig. 6 (noncollocated control) with Fig. 9 (collocated control). Recall that the excitation frequency is $21.2 \mathrm{~Hz}$ in both figures. In the case of noncollocated control, at $21.2 \mathrm{~Hz}$, the harmonic resonance peak is found (see Fig. 2) which corresponds to the set-point $k_{\mathrm{p}}=0 \mathrm{~N} / \mathrm{m}$ and $k_{\mathrm{d}}=$ $0 \mathrm{Ns} / \mathrm{m}$ (no control) in Fig. 6. Due to repositioning of the controller to the midpoint of the beam, the stiffness of the uncontrolled system has increased a little bit, resulting in a small increase of the first harmonic resonance peak to $21.75 \mathrm{~Hz}$. In Fig. 9, the highest max disp value is now found for the set-point $k_{\mathrm{p}}=-5000 \mathrm{~N} / \mathrm{m}$ and $k_{\mathrm{d}}=0 \mathrm{Ns} / \mathrm{m}$. Indeed, some negative $\mathrm{P}$ control is needed to decrease the effective stiffness of the system, in order to find a maximum max disp value again at $21.2 \mathrm{~Hz}$. This can also be seen when comparing the frequencies, where the first harmonic resonance peak occurs in the uncontrolled situation, for the noncollocated case in Fig. 8 and for the collocated case in Fig. 10. After zooming in, it can be observed in Fig. 10 that in the uncontrolled situation (dashed lines) the first harmonic resonance peak has increased a little bit from $21.2 \mathrm{~Hz}$ to $21.75 \mathrm{~Hz}$.

When comparing the results of Figs. 8 and 10 with respect to control objective 2, two differences between the simulations with control (solid lines) can be seen. The first difference occurs near $54 \mathrm{~Hz}$. In the noncollocated case (Fig. 8), the harmonic resonance of the second nonlinear normal mode is clearly visible. This resonance is completely absent in the collocated case (Fig. 10). This is due to the fact that in the col- 
Fig. 10 Vibration reduction in the range 10-60 [Hz]. Solid line: simulation results collocated control (set-point: $k_{\mathrm{p}}=0[\mathrm{~N} / \mathrm{m}]$, $\left.k_{\mathrm{d}}=600[\mathrm{Ns} / \mathrm{m}]\right)$. Dashed line: uncontrolled case. Black dots: unstable numerical solutions of the uncontrolled case

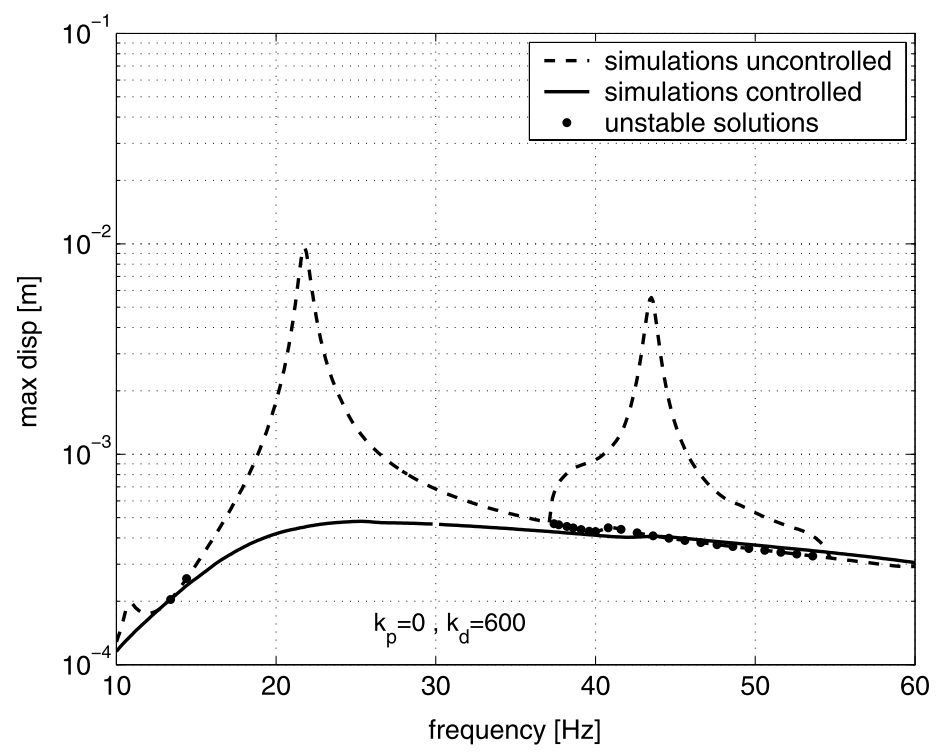

located case, the second nonlinear normal mode actually becomes a linear eigenmode, which has a node at the beam midpoint, due to symmetry in the structure. Therefore, this eigenmode will not be excited by the PD controller force. This eigenmode is nearly equal to the third eigenmode visible in Fig. 3 for the noncollocated case. The second difference is that in the collocated case no $1 / 2$ subharmonic solutions occur anymore; see the solid line in Fig. 10, whereas these still were present in a small frequency range near $38 \mathrm{~Hz}$ for the non-collocated case. See the solid line in Fig. 8.

Note that, apart from the minor differences mentioned above, the global behavior of the controlled system in the frequency range $10-60 \mathrm{~Hz}$ hardly changes by repositioning of the PD controller.

\section{Comparison with passive control}

In [25], passive vibration control of the same piecewise linear beam system was applied by attaching a linear Dynamic Vibration Absorber (DVA) to the beam instead of the PD-controller. The DVA was attached to the beam at exactly the same position as the PDcontroller. The eigenfrequency of this DVA, which basically is a single degree of freedom mass-springdamper system, was tuned to the first harmonic resonance frequency of the piecewise linear system. In [25], the same two control objectives were formulated as in this paper. The undamped DVA was applied to reduce the vibration amplitude at the first harmonic resonance (control objective 1), whereas the damped DVA was used to realize vibration reduction over the frequency range 10-60 [Hz] (control objective 2). The amplitudes of periodic solutions, which are obtained by passive vibration reduction, are shown for control objective 1 in Fig. 11 and for control objective 2 in Fig. 12.

With respect to control objective 1 , it can be seen that the undamped DVA (Fig. 11) realizes a larger vibration reduction at the harmonic resonance frequency than P-control (Fig. 7). When Fig. 7 is compared with Fig. 11, a clear qualitative difference can be noticed. In Fig. 7 (P-control), the minimum at $21.2 \mathrm{~Hz}$ is found at the period doubling bifurcation point between the first harmonic resonance peak and the $1 / 2$ subharmonic resonance peak. In Fig. 11, the minimum at $21.2 \mathrm{~Hz}$ is found at the antiresonance between two harmonic resonance peaks indicated by the two 1's. This difference can be explained by comparing the natures of the active P-controller and the passive, undamped Dynamic Vibration Absorber (DVA). The P-controller only changes the effective stiffness of the controlled structure and, therefore, it basically shifts the resonance frequencies; see Sect. 6. The undamped DVA is a mass-spring system, which in contrast to the active controller, adds an extra degree of freedom and, therefore, an extra harmonic resonance to the system. If the eigenfrequency of the undamped DVA is tuned to the first harmonic resonance frequency of the sys- 
Fig. 11 Suppression of the first harmonic resonance undamped DVA. Solid lines: simulation results DVA, circles: experimental results DVA. Dashed lines: simulation results without DVA peak near 19 [Hz] using an

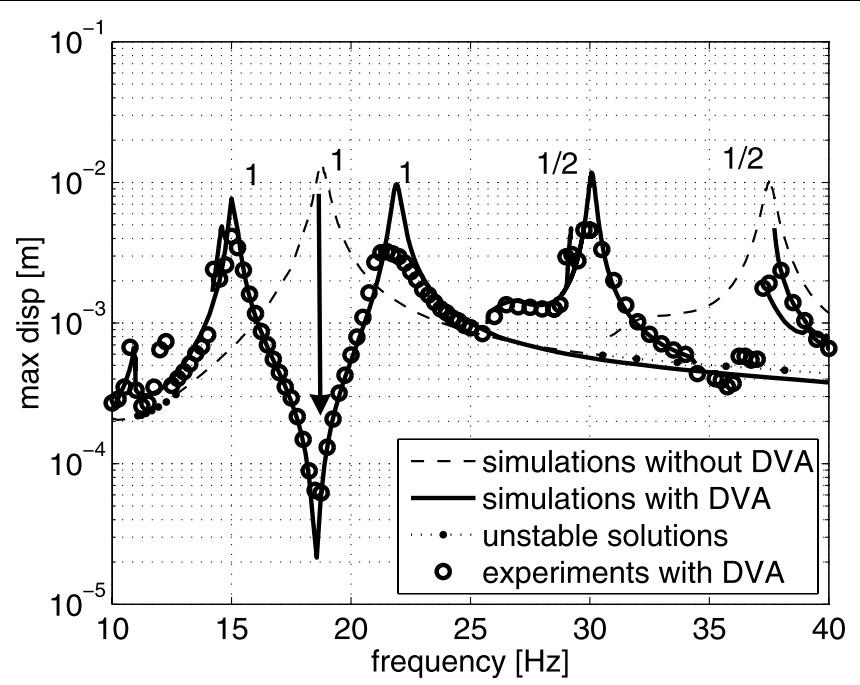

Fig. 12 Vibration reduction in the frequency range $10-60[\mathrm{~Hz}]$ using a damped DVA. Solid lines: simulation results DVA, circles: experimental results DVA. Dashed lines: simulation results without DVA

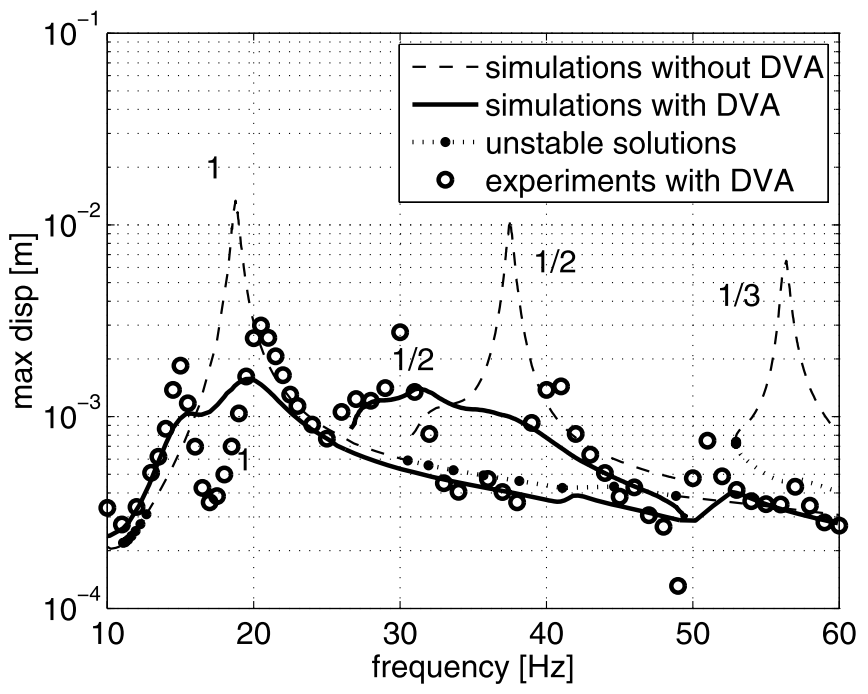

tem without DVA, for the system with DVA at this frequency an anti-resonance results, which is located in between two nearby harmonic resonance peaks. This is completely comparable to the case of an undamped DVA applied to a linear single degree of freedom system as studied in [1].

With respect to control objective 2, the results obtained with D-control (Fig. 8) are apparently superior to the results realized by the damped DVA (Fig. 12). Also here, the natures of the active Dcontroller and the passive, damped DVA are different. The D-controller only changes the effective damping of the controlled structure (see Sect. 6) and, therefore, it basically reduces the resonance amplitudes. Also in the case of the damped DVA, which is a mass-damperspring system, an extra degree of freedom is added to the system. The damping constant of the damped DVA should be chosen such that the maximum value of the amplitudes of the two nearby harmonic resonances is minimized. The solid line in Fig. 12 represents this situation. In the experiments a nonoptimal damping constant was used (circles in Fig. 12) resulting in higher amplitude values for the two nearby harmonic resonances. In both the actively controlled case and in the passively controlled case, amplitude reduction of the harmonic resonance peaks also results in amplitude reduction of the related $1 / 2$ subharmonic resonances. 


\section{Conclusions and recommendations}

This paper shows that a linear PD-controller can be used very effectively for vibration reduction of piecewise linear systems, for the considered case of flush with a moderate amount of nonlinearity. Simulation results have been validated by experimental results and in almost all cases a good correspondence has been obtained. The effects of P-action and D-action have been investigated separately. Proportional feedback mainly shifts the frequencies where (sub)harmonic resonances occur. In this paper, negative $\mathrm{P}$-action has been used very effectively to decrease the vibration level at the frequency, where the first harmonic resonance peak occurs in the uncontrolled system, by shifting this resonance peak to a lower frequency. Application of D-action results in substantial reduction of the resonance amplitudes in the whole frequency range of interest, ranging from 10 to $60[\mathrm{~Hz}]$.

For the case of flush considered in this paper, the results indicate that the effect of PD-control applied on a piecewise linear system to a large extent is comparable to the effect of PD-control applied on a linear system.

The performances of the active PD-controller and the passive DVA have been compared for the cases considered. With respect to the first control objective, the undamped DVA was superior to the active P-controller. With respect to the second control objective, the active D-controller performed better than the damped DVA. However, a general objective tradeoff between PD-control and a tuned DVA for effectiveness in vibration reduction is difficult to make, because many factors will have to be taken into account: costs, controller weight, controller power, ease of implementation, control objectives, etc. In general, the importance of certain issues may differ from situation to situation.

The vibration reduction, which is realized for the transversal displacement of the midpoint of the beam, does not guarantee vibration reduction for transversal displacements of other positions on the beam. However, by visual inspection, it was verified that also on other beam positions vibration reduction is achieved, except for excitation frequencies near the second harmonic resonance peak.

A direction for future research will be the issue of the uniqueness of the steady-state solutions found for the controlled cases, because a proof of uniqueness would further increase the value of the results obtained in this paper. Theory for proving uniqueness of periodic solutions has been developed in [37] and may be applied to the periodic solutions found for the piecewise linear system studied in the current paper.

Open Access This article is distributed under the terms of the Creative Commons Attribution Noncommercial License which permits any noncommercial use, distribution, and reproduction in any medium, provided the original author(s) and source are credited

\section{References}

1. den Hartog, J.P.: Mechanical Vibrations, 4th edn. Dover, New York (1985)

2. Hunt, J.B.: Dynamic Vibration Absorbers. Mechanical Engineering Publications, London (1979)

3. Korenev, B.G., Reznikov, L.M.: Dynamic Vibration Absorbers. Wiley, New York (1993)

4. Mead, D.J.: Passive Vibration Control. Wiley, New York (1999)

5. Preumont, A.: Vibration Control of Active Structuresan Introduction, 2nd edn. Kluwer Academic, Dordrecht (2002)

6. Franklin, G.F., Powell, J.D., Emami-Naeni, A.: Feedback Control of Dynamic Systems, 5th edn. Pearson, Prentice Hall, Upper Saddle River, New Jersey (2006)

7. Gawronski, W.K.: Advanced Structural Dynamics and Active Control of Structures. Springer, New York (2004)

8. Meirovitch, L.: Dynamics and Control of Structures. Wiley, New York (1990)

9. Preumont, A., Seto, K.: Active Control of Structures. Wiley, New York (2008)

10. van de Vorst, E.L.B., Assinck, F.H., de Kraker, A., Fey, R.H.B., van Campen, D.H.: Experimental verification of the steady-state behaviour of a beam system with discontinuous support. Exp. Mech. 36(2), 159-165 (1996)

11. Thompson, J.M.T., Elvey, J.S.N.: Elimination of subharmonic resonances of compliant marine structures. Int. J. Mech. Sci. 26(6-8), 419-426 (1984)

12. Fey, R.H.B., van Liempt, F.P.H.: Sine sweep and steadystate response of simplified solar array models with nonlinear elements. In: Maia N.M.M., Montalvao e Silva J.M., Relogio Ribeiro A.M. (eds.) Proceedings of the Int. Conf. on Structural Dynamics Modeling, Funchal, Madeira, Portugal, June 3-5, pp. 201-210 (2002)

13. de Freitas, M.S.T., Viana, R.L., Grebogi, C.: Erosion of the safe basin for the transversal oscillations of a suspension bridge. Chaos Solitons Fractals 18, 829-841 (2003)

14. Drugge, L., Larsson, T., Berghuvud, A., Stensson, A.: The nonlinear behaviour of a pantograph current collector suspension. In: Proceedings of the 1999 ASME DETC, Las Vegas NV, September 12-15, paper DETC99/VIB-8026 (1999)

15. Jerrelind, J., Stensson, A.: Nonlinear dynamics of parts in engineering systems. Chaos Solitons Fractals 11, 2413$2428(2000)$ 
16. Stensson, A., Asplund, C., Karlsson, L.: The nonlinear behavior of a MacPherson strut wheel suspension. Veh. Syst. Dyn. 23, 85-106 (1994)

17. Fey, R.H.B., van Campen, D.H., de Kraker, A.: Long term structural dynamics of mechanical systems with local nonlinearities. ASME J. Vib. Acoust. 118(2), 147-153 (1996)

18. Shaw, S.W., Holmes, P.J.: A periodically forced piecewise linear oscillator. J. Sound Vib. 90(1), 129-155 (1983)

19. Jiang, D., Pierre, C., Shaw, S.W.: Nonlinear normal modes for vibratory systems under harmonic excitation. J. Sound Vib. 288, 791-812 (2005)

20. Luo, A.C.J., Menon, S.: Global chaos in a periodically forced, linear system with a dead zone restoring force. Chaos Solitons Fractals 19, 1189-1199 (2004)

21. Wiercigroch, M., Sin, V.W.T., Li, K.: Measurement of chaotic vibration in a symmetrically piecewise linear oscillator. Chaos Solitons Fractals 9(1-2), 209-220 (1998)

22. Ervin, E.K., Wickert, J.A.: Experiments on a beam-rigid body structure repetitively impacting a rod. Nonlinear Dyn. 50, 701-716 (2007)

23. Natsiavas, S.: Periodic response and stability of oscillators with symmetric trilinear restoring force. J. Sound Vib. 134(2), 315-331 (1989)

24. Shukla, A., Besselink, B., Fey, R.H.B., Nijmeijer, H.: Classification of periodic orbits for systems with backlash. Chaos Solitons Fractals 41, 131-144 (2009)

25. Bonsel, J.H., Fey, R.H.B., Nijmeijer, H.: Application of a dynamic vibration absorber to a piecewise linear system. Nonlinear Dyn. 37(3), 227-243 (2004)

26. Doris, A.: Output-feedback design for non-smooth mechanical systems: control synthesis and experiments. Ph.D. thesis, Eindhoven University of Technology (2007)

27. Doris, A., Juloski, A.L., Mihajlovic, N., Heemels, W.P.M.H., van de Wouw, N., Nijmeijer, H.: Observer de- signs for experimental non-smooth and discontinuous systems. IEEE Trans. Control Syst. Technol. 16(6), 1323-1332 (2008)

28. Heertjes, M.F., van de Molengraft, M.J.G., Nijmeijer, H.: Partial feedback linearization on a harmonically excited beam with a one-sided spring. J. Sound Vib. 228(5), 939959 (1999)

29. Heertjes, M.F., van de Molengraft, M.J.G.: Controlling the nonlinear dynamics of a beam system. Chaos Solitons Fractals 12, 49-66 (2001)

30. Parker, T.S., Chua, L.O.: Practical Numerical Algorithms for Chaotic Systems. Springer, New York (1989)

31. Thomsen, J.J.: Vibrations and Stability, Advanced Theory, Analysis, and Tools, 2nd edn. Springer, New York (2003)

32. Leine, R.I., Nijmeijer, H.: Dynamics and Bifurcations of Non-smooth Mechanical Systems. LNAMC, vol. 18. Springer, Berlin (2004)

33. Wiercigroch, M.: Modelling of dynamical systems with motion dependent discontinuities. Chaos Solitons Fractals 11, 2429-2442 (2000)

34. Touzé, C., Amabili, M.: Nonlinear normal modes for damped geometrically nonlinear systems: Application to reduced-order modelling of harmonically forced structures. J. Sound Vib. 298, 958-981 (2006)

35. Vakakis, A.F.: Nonlinear normal modes and their applications in vibration theory: an overview. Mech. Syst. Signal Process. 11, 3-22 (1997)

36. Craig, R.R.Jr., Kurdila, A.J.: Fundamentals of Structural Dynamics, 2nd edn. Wiley, New York (2006)

37. Pavlov, A., Wouw, N., van de Nijmeijer, H.: Uniform Output Regulation of Nonlinear Systems: A Convergent Dynamics Approach. Series: Systems \& Control: Foundations \& Applications. Birkhäuser, Basel (2006) 